\title{
The homeodomain protein $h m b x-1$ maintains asymmetric gene expression in adult C. elegans olfactory neurons
}

\author{
Bluma J. Lesch and Cornelia I. Bargmann ${ }^{1}$ \\ Howard Hughes Medical Institute, Laboratory of Neural Circuits and Behavior, The Rockefeller University, New York,
} New York 10065, USA

Differentiated neurons balance the need to maintain a stable identity with their flexible responses to dynamic environmental inputs. Here we characterize these opposing influences on gene expression in Caenorhabditis elegans olfactory neurons. Using transcriptional reporters that are expressed differentially in two olfactory neurons, $A \mathrm{CC}^{\mathrm{ON}}$ and $\mathrm{AWC} \mathrm{OFF}^{\mathrm{O}}$, we identify mutations that affect the long-term maintenance of appropriate chemoreceptor expression. A newly identified gene from this screen, the conserved transcription factor hmbx-1, stabilizes AWC gene expression in adult animals through dosage-sensitive interactions with its transcriptional targets. The late action of $h m b x-1$ complements the early role of the transcriptional repressor gene $n s y-7$ : Both repress expression of multiple $\mathrm{AWC} \mathrm{OFF}^{\mathrm{OF}}$ genes in $\mathrm{AWC}^{\mathrm{ON}}$ neurons, but they act at different developmental stages. Environmental signals are superimposed onto this stable cell identity through at least two different transcriptional pathways that regulate individual chemoreceptor genes: a cGMP pathway regulated by sensory activity, and a daf-7 (TGF- $\beta$ )/daf-3 (SMAD repressor) pathway regulated by specific components of the density-dependent C. elegans dauer pheromone.

[Keywords: Chemoreceptor, olfactory receptor; homeostasis; neuronal development; olfactory neuron]

Supplemental material is available at http://www.genesdev.org.

Received March 31, 2010; revised version accepted June 25, 2010.

Neurons and other long-lived cells are subject to ongoing modification throughout life, but use transcriptional strategies to maintain stable cell fates (Shirasaki and Pfaff 2002; Ringrose and Paro 2004). In some cells, transcription factors required for the establishment of cell fate remain active throughout life. In the mammalian immune system, the transcription factor Pax 5 is required both for initial commitment to the B-cell lineage and for continued expression of B-cell identity: Deletion of Pax5 from committed pro-B cells resulted in their reversion to a multipotential state (Nutt et al. 1999; Mikkola et al. 2002). In other cases, dedicated transcriptional regulators maintain cell identity. For example, the Caenorhabditis elegans aristaless homolog alr-1 is required to maintain, but not establish, functions of sensory glia (Tucker et al. 2005). In addition to cell-specific transcription factors, general chromatin remodeling factors can stabilize cell fates. The Polycomb group (PcG) genes in Drosophila maintain a precise Hox gene expression pattern after the disappearance of early developmental regulators (Busturia and Morata 1988; Cao et al. 2002; Muller and Kassis

${ }^{1}$ Corresponding author.

E-MAIL cori@rockefeller.edu; FAX (212) 327-7243.

Article is online at http://www.genesdev.org/cgi/doi/10.1101/gad.1932610.
2006), and the histone acetyltransferase system regulated by BET-1 and MYST maintains cell type-specific gene expression in C. elegans neuronal lineages (Shibata et al. 2010).

Sensory neurons are faced with the special challenge of maintaining a stable state while responding to a changing environment. In the senses of taste and smell, heterogeneous populations of sensory neurons express different chemoreceptor genes to detect different environmental chemicals (Buck and Axel 1991; Troemel et al. 1995; Vosshall et al. 1999; Clyne et al. 2000; Etchberger et al. 2007). The expression of chemoreceptor genes is initiated by innate developmental programs. For example, in $C$. elegans, chemoreceptor expression in the two ASE taste neurons is initiated by a general transcriptional regulator for ASE, che-1, and refined by a double negative feedback loop that distinguishes right and left ASE fates /Chang et al. 2003, 2004; Johnston and Hobert 2003; Johnston et al. 2005; Etchberger et al. 2007, 2009). che-1 maintains its own expression in the ASE chemosensory neurons, and also acts on target genes throughout life (Etchberger et al. 2009); this combination of initiation and differentiation roles defines che-1 as a "terminal selector gene" (Hobert 2008). Analogous genetic studies of other chemosensory cell types-including the AWA, AWB, and AWC 
olfactory neurons-have generated a sophisticated understanding of the transcription factors that initiate unique neuronal identities (Sengupta et al. 1994; Sagasti et al. 1999; Sarafi-Reinach and Sengupta 2000; SarafiReinach et al. 2001; Colosimo et al. 2003; Lanjuin et al. 2003; Nokes et al. 2009; Kim et al. 2010).

Superimposed on stable chemosensory neuron fates are environmental factors that modify gene expression. The C. elegans dauer pheromone, a mixture of compounds containing the sugar ascarylose, represses the expression of chemoreceptor genes in ASH and ASI neurons by regulating intercellular signaling through a TGF- $\beta$ signaling pathway (Peckol et al. 2001; Nolan et al. 2002; Kim et al. 2009). A salt-inducible kinase affects chemoreceptor expression in AWB olfactory neurons by regulating the transcription factor MEF2 (Lanjuin and Sengupta 2002; van der Linden et al. 2007, 2008). The relationship between these environmental regulators and stable cell fates raises intriguing questions about the relative roles of fixed and variable aspects of neuronal function.

The two C. elegans AWC olfactory neurons provide a system in which the acquisition and maintenance of cell fates are distinct. The Otx transcription factor CEH36 and the HMX/NLX homeodomain protein MLS-2 initiate a general AWC identity, which is subsequently maintained by ceh-36 (Lanjuin et al. 2003; Kim et al. 2010). In addition to promoting the expression of an AWC-specific transcriptional program, ceh-36 maintains its own expression, suggesting that ceh-36 is the terminal selector gene in AWC (Kim et al. 2010). Later in embryogenesis, a stochastic cell fate decision causes the right and left AWC olfactory neurons to take on asymmetric fates, such that one $\mathrm{AWC}$ becomes $\mathrm{AWC}^{\mathrm{ON}}$, defined as a neuron that expresses the G protein-coupled receptor (GPCR) str-2 and senses the odor butanone, and the other AWC becomes AWC ${ }^{\mathrm{OFF}}$, which expresses the GPCR srsx-3 and senses the odor 2,3-pentanedione (Troemel et al. 1999; Wes and Bargmann 2001). The decision to become $\mathrm{AWC}^{\mathrm{ON}}$ or $\mathrm{AWC} \mathrm{OFF}^{\mathrm{OF}}$ is made through a signaling pathway that generates the initial asymmetry of chemoreceptor gene expression and also drives asymmetric expression of the transcription factor NSY-7 in $\mathrm{AWC}^{\mathrm{ON}}$ (Troemel et al. 1999; Lesch et al. 2009). After hatching, the initial signaling pathway becomes inactive, and NSY-7 maintains appropriate chemoreceptor expression in $\mathrm{AWC}^{\mathrm{ON}}$. In addition, a cGMP pathway regulated by olfactory signal transduction maintains post-embryonic expression of both str-2 and srsx-3 using two receptor-type guanylate cyclases (encoded by odr-1 and daf-11), a cyclic-nucleotide gated cation channel (encoded by tax-2 and tax-4), and a cGMP-responsive protein kinase (encoded by egl-4) (Troemel et al. 1999; Lesch et al. 2009).

To better understand the factors responsible for the stability of $\mathrm{AWC}^{\mathrm{ON}}$ and $\mathrm{AWC}^{\mathrm{OFF}}$ fates, we performed a screen for mutants that failed to maintain expression of one or both of the asymmetric AWC markers str-2 and srsx-3. Here, we describe the mutants isolated from this screen. We identify the transcription factor $h m b x-1$, a homolog of mammalian HMBOX1, as a regulator of AWC receptor gene expression that acts primarily in adult animals. Using newly identified GPCR genes expressed in AWC neurons, we show that the maintenance of asymmetric receptor gene expression involves at least three repressor pathways: $n s y-7$, an $\mathrm{AWC}^{\mathrm{ON}}$-specific cell identity gene; $h m b x-1$, a dosage-sensitive repressor of $\mathrm{AWC}^{\mathrm{OFF}}$ genes in $\mathrm{AWC}^{\mathrm{ON}}$ neurons; and daf-3, a pheromone-regulated repressor that affects chemoreceptors on a gene-by-gene basis.

\section{Results}

Genetic pathways required for maintenance of GPCR expression in $A W C$

We sought mutants that expressed the $\mathrm{AWC}^{\mathrm{ON}}$-specific receptor str-2 and the $\mathrm{AWC}^{\mathrm{OFF}}$-specific receptor srsx-3 appropriately as early larvae, but failed to maintain expression of one or both of these receptors in adulthood. A strain with an integrated transgene containing str$2:: d s R e d 2$ and srsx $-3::$ GFP reporters was mutagenized, and the adult F2 progeny were examined for defects in expression of str-2, srsx-3, or both genes (Fig. 1A). After verifying the adult phenotype in subsequent generations, expression of str-2 and srsx-3 was evaluated in early larvae, $14 \mathrm{~h}$ after hatching (L1 stage), and mutants with a wild-type phenotype at this stage were retained. The screen yielded 19 mutants with defects in the maintenance of AWC markers (Fig. 1B). Genetic mapping and complementation testing indicated that 13 mutations fell in previously characterized genes and pathways, although only a subset had been known to affect AWC gene expression. These genes are described briefly below.

Olfactory transduction Six of the new mutations affect the olfactory cGMP transduction pathway that maintains str-2 and srsx-3 expression, including three alleles of odr-1, one allele of daf-11, one allele of tax-2, and one allele of tax-4 (Fig. 1B; Troemel et al. 1999; Lesch et al. 2009).

Previous studies have revealed both cell-autonomous and nonautonomous effects of sensory signaling proteins on AWC gene expression (Lans and Jansen 2006). Moreover, tax-4 promotes expression of daf-7 (Coburn et al. 1998), which acts in ASI neurons to promote srsx-3 expression in AWC neurons (see below). To ask where tax -4 acts to regulate srs $x-3$ expression, we expressed the tax-4 cDNA in AWC or ASI in a tax-4(ky791) mutant background. AWC-selective expression of TAX-4 rescued the srsx-3 expression defect of tax-4(ky791) mutants, but ASI expression did not (Supplemental Fig. S1). Therefore, tax-4 acts in AWC to promote srs $x-3$ expression.

The screen also yielded a dominant mutation in the $\mathrm{G} \alpha$ subunit odr-3, odr-3(ky879), which encodes a G $\rightarrow$ S missense mutation at position 185 . The affected glycine is a conserved residue in the region that changes conformation upon GTP binding; stabilization of the GTPbound conformation should result in a constitutively active protein (Rens-Domiano and Hamm 1995). The nature of the odr-3(ky879) allele suggests that increased olfactory $G$ protein activity disrupts maintenance of 
A

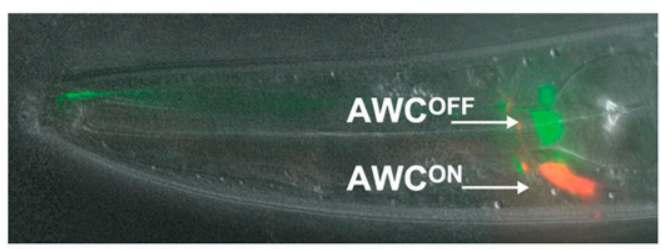

B

\begin{tabular}{|c|c|c|c|c|c|c|c|}
\hline & str-2::c & dsRed & & srsx-3:: & $:$ :GFP & & \\
\hline genotype & 2 on $\begin{array}{c}1 \text { on, } \\
\text { bright }\end{array}$ & $\begin{array}{l}1 \text { on, } \\
\text { faint }\end{array}$ & 2 off & 2 on $\begin{array}{c}1 \text { on, } \\
\text { bright }\end{array}$ & $\begin{array}{l}1 \text { on, } \\
\text { faint }\end{array}$ & 2 off & $n$ \\
\hline & ০০ ০০ & ০০ & ০o & ০০ ০০ & ০০ & ০০ & \\
\hline
\end{tabular}

\begin{tabular}{|c|c|c|c|c|c|c|c|c|c|}
\hline $\begin{array}{l}\text { Group I: } \\
\text { srsx-3::GFP defective }\end{array}$ & & & & & & & & & - \\
\hline $\begin{array}{l}\operatorname{tax}-2(\text { (ky } 782) \\
\text { N329K, channel }\end{array}$ & 1 & 85 & 4 & 10 & 0 & 0 & 4 & 96 & 210 \\
\hline $\begin{array}{l}\operatorname{tax}-4(\mathrm{ky} 791) \\
\text { Q510STOP, after tm domain }\end{array}$ & 1 & 70 & 13 & 16 & 0 & 0 & 10 & 90 & 286 \\
\hline $\begin{array}{l}\text { daf-7(ky771) } \\
\text { Q105STOP }\end{array}$ & 0 & 97 & 0 & 3 & 0 & 0 & 5 & 95 & 66 \\
\hline $\begin{array}{l}\text { daf-1 (ky803) } \\
\text { G400E, kinase }\end{array}$ & 0 & 73 & 0 & 27 & 0 & 0 & 0 & 100 & 44 \\
\hline unc-3(ky793) & 0 & 98 & 2 & 0 & 0 & 0 & 0 & 100 & 65 \\
\hline $\begin{array}{c}\text { hmbx-1(ky777) } \\
\text { H404Y }\end{array}$ & 0 & 100 & 0 & 0 & 0 & 0 & 1 & 99 & 199 \\
\hline ky 811 & 0 & 100 & 0 & 0 & 0 & 0 & 7 & 93 & 144 \\
\hline
\end{tabular}

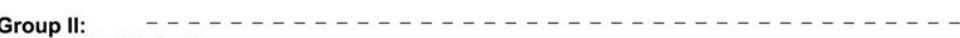

str-2::dsRed defective

$\begin{array}{lccccccccc}\begin{array}{l}n s y-7(k y 813) \\ n s y-7(k y 819) \\ \text { tam-1(ky798) }\end{array} \quad 0 & 0 & 0 & 100 & 98 & 0 & 0 & 2 & 53 \\ \quad \text { G76S, zinc finger } & 0 & 0 & 27 & 73 & 100 & 0 & 0 & 0 & 64 \\ \begin{array}{l}\text { ky789 } \\ \text { ky802 }\end{array} & 0 & 8 & 24 & 68 & 0 & 95 & 5 & 0 & 248 \\ & 0 & 0 & 11 & 89 & 3 & 97 & 0 & 0 & 38 \\ & 0 & 0 & 0 & 100 & 0 & 92 & 8 & 0 & 52\end{array}$

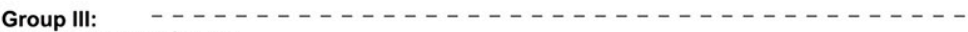
both markers defective

\begin{tabular}{|c|c|c|c|c|c|c|c|c|c|}
\hline odr-1(ky779) & 0 & 0 & 6 & 94 & 0 & 0 & 0 & 100 & 51 \\
\hline odr-1(ky800) & 0 & 0 & 9 & 91 & 0 & 1 & 1 & 98 & 89 \\
\hline odr-1(ky807) & 0 & 0 & 66 & 34 & 0 & 0 & 2 & 98 & 99 \\
\hline daf-11(ky801) & 0 & 1 & 36 & 63 & 0 & 21 & 42 & 36 & 184 \\
\hline $\begin{array}{l}\text { D808V, cyclase domain } \\
\text { odr-3(ky879) } \\
\text { G185S, GTP binding }\end{array}$ & 0 & 9 & 16 & 74 & 0 & 61 & 23 & 17 & 160 \\
\hline ky772 & 0 & 2 & 58 & 40 & 2 & 2 & 96 & 0 & 43 \\
\hline ky 773 & 0 & 0 & 12 & 88 & 0 & 0 & 100 & 0 & 52 \\
\hline
\end{tabular}

known alleles odr-1(n1936)

daf-11(m47)

tax-2(ks31)

$\operatorname{tax}-4($ (p678)

nsy-7(tm3080)

tam-1(cc567)

daf-7(e1372)

daf- $1(m 40)$

$\begin{array}{lccc}0 & 0 & 18 & 82 \\ 0 & 0 & 4 & 96 \\ 0 & 94 & 3 & 3 \\ 0 & 90 & 10 & 0 \\ 0 & 0 & 0 & 100 \\ 0 & 86 & 12 & 2 \\ 0 & 100 & 0 & 0 \\ 0 & 100 & 0 & 0\end{array}$

GPCR expression, perhaps by reducing cGMP levels (Chalasani et al. 2007). Loss-of-function mutations in odr-3 have little effect on str-2 expression, although str-2 expression is reduced when odr-3(lf) alleles are combined with mutations in other G $\alpha$ subunits (Lans and Jansen 2006).

Transcriptional regulation Two alleles of nsy-7 isolated in the screen had defective maintenance of str-2 expression, accompanied by bilateral srs $x-3$ expression-the
Figure 1. Expression phenotypes of mutants defective for str-2::dsRed and srsx-3::GFP maintenance. (A) DIC image of the head of an adult worm overlaid with a fluorescence image of the str-2::dsRed2 $\left(\mathrm{AWC}^{\mathrm{ON}}\right)$ and srs $\mathrm{x}-3:: G F P\left(\mathrm{AWC}^{\mathrm{OFF}}\right)$ reporters. (B) Mutants from the screen. (Group I) srs $x-3$ expression affected more strongly than str-2 expression. (Group II) str-2 expression affected more strongly than srsx-3 expression. (Group III) Both markers affected. Other alleles of genes identified in the screen are shown for reference. Bright and faint fluorescence were scored qualitatively; in other figures, these two categories are combined into a single "1 AWC" class. 
was not known previously to affect gene expression in AWC, but diminished expression of the AWC reporter genes is consistent with the general reduction in transgene expression reported in tam-1 mutants.

$D A F-7 / T G F \beta$ signaling Several mutations from this screen primarily affected srs $x-3$ and not str-2 expression. These included an allele of daf-7 (the TGF $\beta$ ligand that regulates the dauer developmental decision and chemoreceptor gene expression in ASH and ASI neurons) and an allele of daf-1 (a TGF $\beta$ type I receptor) (Figs. 1B, 2A,B; Georgi et al. 1990; Ren et al. 1996; Schackwitz et al. 1996; Peckol et al. 2001; Nolan et al. 2002). A mutation in unc-3, a transcription factor required for expression of daf-7 in the ASI neurons, was also isolated in the screen, and had a similar phenotype (Prasad et al. 1998; Kim et al. 2005). Existing daf-4 (the type II TGF $\beta$ receptor), daf- 8 (a Smadlike protein), and daf-14 (a Smad-like protein) mutants displayed similar adult phenotypes to daf-7 and daf-1 (Fig. $2 \mathrm{C})$. These results indicate that TGF $\beta$ signaling maintains srs $x-3$ expression in $\mathrm{AWC}^{\mathrm{OFF}}$.

daf-3, which encodes a co-Smad that binds DNA, and daf-5, which encodes a proline-rich transcriptional regulator, act downstream from and antagonistically to daf-1 to promote dauer formation (Patterson et al. 1997; da Graca et al. 2004). Expression of str-2 and srsx-3 reporters was normal in daf-3 and daf-5 single mutants, and in daf-3; daf-1 or daf-5; daf-1 double mutants, recapitulating the regulatory relationships seen in dauer formation (Fig. 2B,C). Expression of DAF-1 in AWC rescued the srs $x-3$ expression defect of daf-1(m40) mutants, suggesting that TGF- $\beta$ signals directly to AWC to maintain receptor gene expression (Fig. 2C).

daf-7 expression is inhibited by the dauer pheromone, a mixture of structurally related chemicals termed ascarosides (Jeong et al. 2005; Butcher et al. 2007, 2008). To ask whether srsx-3 expression responded acutely to pheromones, we exposed adult worms to the ascarosides C3, C6, and C9 for $4 \mathrm{~h}$, and monitored expression of srs $\mathrm{x}-3$ using a destabilized GFP protein that has a half-life of $\sim 1$ $\mathrm{h}$ in C. elegans (Gaudet and Mango 2002; Frand et al. 2005). An equal mixture of C3, C6, and C9 suppressed srsx-3 expression in a dose-dependent manner (Fig. 2D). The ascarosides C3 and C6 each suppressed srsx-3 expression about as well as the mixture, while C9 was less effective. All effects of ascarosides were blocked in daf-3 mutants, suggesting that $\mathrm{C} 3$ and $\mathrm{C} 6$ pheromones regulate srs $x-3$ expression through the TGF $\beta$ pathway (Fig. 2D).
A

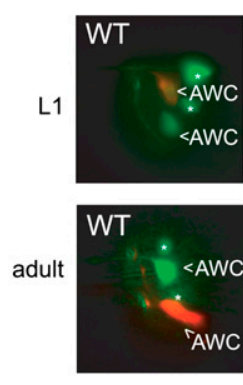

C

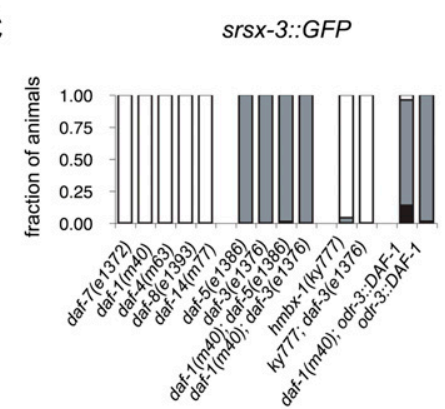

B

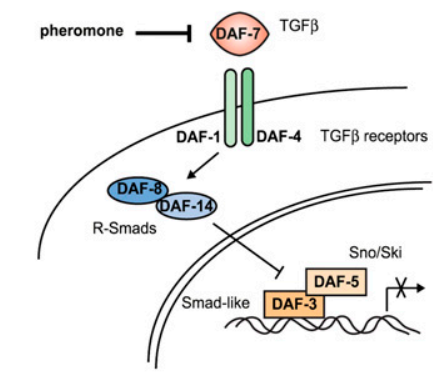

str-2::DsRed
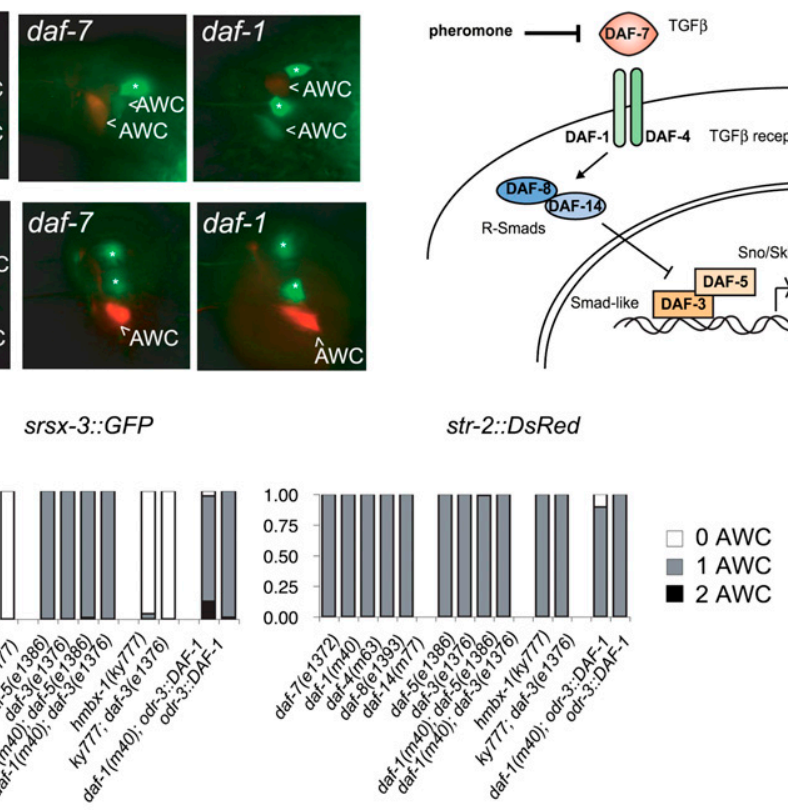

D
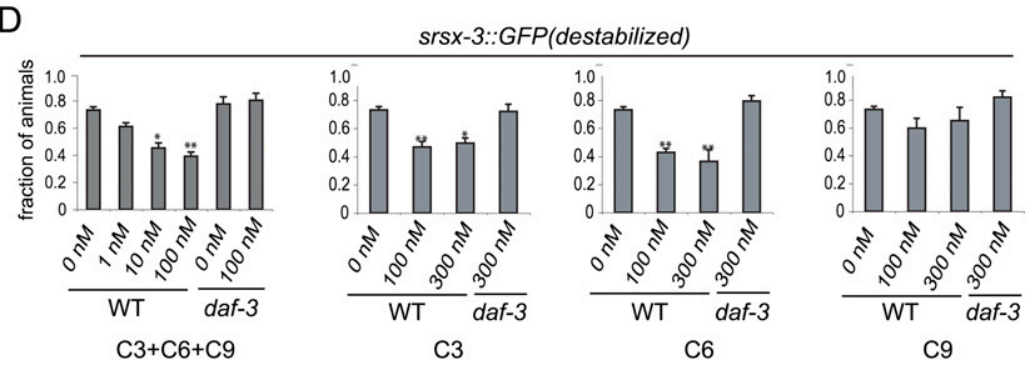

Figure 2. TGF $\beta$ and dauer pheromone signals regulate srsx-3 expression. (A) Images of L1 larvae and adults in wild-type, daf-7(e1372), and daf-1(m40) animals. AWC neurons are labeled, and asterisks mark AWB neurons. (B) Schematic illustration of the daf-7 TGF $\beta$ pathway. $(C)$ srsx-3 and str-2 expression phenotypes for $d a f-7 /$ TGF- $\beta$ pathway mutants and rescue of daf-1 $(\mathrm{m} 40)$ under the AWC-selective promoter $o d r-3$. Also shown is the phenotype of the $h m b x-$ 1(ky777); daf-3(e1376) double mutant. $n>25$ for all genotypes. $(D)$ Regulation of srsx-3 by a mixture of C3, C6, and C9 ascarosides (left) and by each ascaroside alone (two middle and right). Graphs show the fraction of adults expressing a destabilized GFP in AWC under the control of the srsx-3 promoter. $\left(^{\star}\right) P<0.01 ;\left(^{* *}\right) P<0.001$ compared with no pheromone control (one-way ANOVA with Dunnett's post-test). 
A missense allele of the transcription factor hmbx-1 suppresses srsx-3 expression

In the mutant ky777, srsx-3 expression in $\mathrm{AWC}^{\mathrm{OFF}}$ was lost after L1, but str-2 expression in $\mathrm{AWC}^{\mathrm{ON}}$ was retained (Figs. 1, 3A). Although this phenotype resembled that of

A

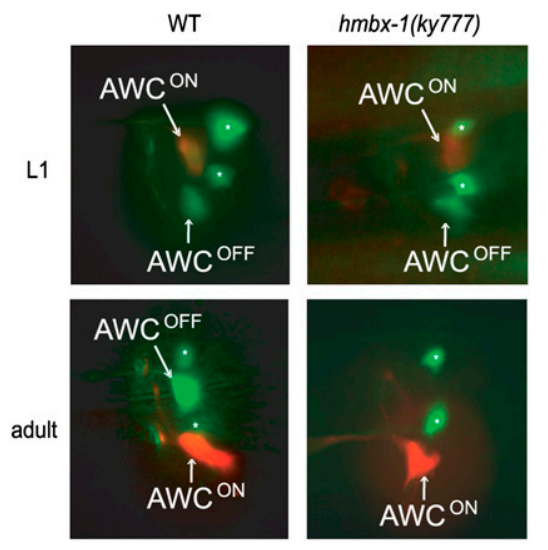

B

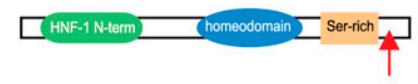

MLFTIEQLELIKKLQHTGMSSDQLLKAFGELEVPEQLQNNNTIAAALYSPLLVQHLTTPK SETPVKLTVQTVPTPVKSEPQSSNCSSPFEHPICSNAPRPIRSQRTPMKEITTLDDPNEL EEFMKQGEEACILDMKTFITQYSLRQTTVAMMTGVVSPYISKLLNGNHRELSLRCRKNIY CWYLNCRRHPNKLAAFLADPTTRLETNGDGELIPORRERYVFRPILIRMLESFFTOTPFP DLPRRVEIANAdNHVLKMDKKGVGLMPKEVVPQVWVNWFANKRKELRRRSAEASAASTS SASSSASSTANHDSVSVSSMSPRDEETSSRNTTPETAISPSPAVSTFEVSRPSAIISATS STTSPISIPATIIPSVSPSALELFAMAQQLGVQLPVFPTLPTH/HFPFQMAPFYGNPASI LKSE$\mathrm{H} 404 \mathrm{Y}$

\section{C}

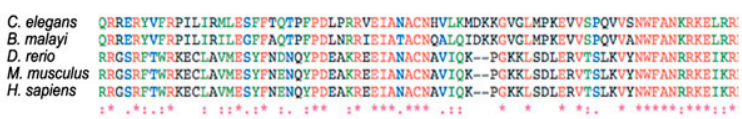

D

E
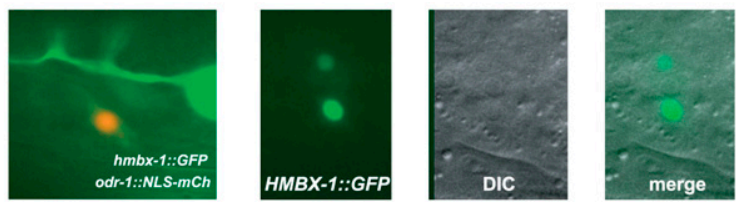

Figure 3. ky777 is an allele of the homeodomain transcription factor hmbx-1. (A) Fluorescence images of early larval and adult wild-type and ky777 mutant animals showing late loss of srsx$3:: G F P$ from $\mathrm{AWC}^{\mathrm{OFF}}$. AWC neurons are labeled, and asterisks mark AWB neurons. $(B, t o p)$ Domain structure and amino acid sequence of HMBX-1. Red arrow indicates the location of the ky777 mutation in the domain structure diagram. (Bottom) The ky777 mutation is boxed in red, the beginning of the $\operatorname{tm} 1274$ deletion is indicated with a red bracket, the homeodomain is underlined, and a 17-amino-acid insertion in the homeodomain is boxed in black. $(C)$ Conservation of the HMBX-1 homeodomain. (D) Expression of $h m b x-1$ (promoter) ::GFP in AWC. AWC is marked by a nuclear-localized mCherry reporter under the control of the odr-1 promoter. Also visible are parts of the anterior process and cell body of the FLP neuron. (E) Nuclear localization of HMBX-1::GFP fusion protein in AWC. GFP is restricted to nuclei, visualized by DIC microscopy.
daf-7/TGF $\beta$ mutants, ky777 mutants were normal for dauer formation, and the srsx-3 expression defect in these mutants was not suppressed by daf-3 (Fig. 2C). Therefore, ky777 appeared to act separately from the TGF $\beta$ pathway. ky777 was mapped to an interval of $\sim 440 \mathrm{~kb}$ on the left arm of chromosome I, but no rescue was observed after injection of fosmids or PCR products covering the interval. Therefore, a Solexa-Illumina whole-genome sequencing approach was used to identify mutations in the interval. Unique alignments of ky777 sequence reads to the C. elegans genome accounted for $84 \%$ of sequence in the interval, with an average coverage depth of $7.9 \times$. The gigaBayes program identified 19 high-probability point mutations and three single-base indels between ky777 and the reference genome. Two missense mutations and one silent mutation were present in coding exons, one mutation was in a $3^{\prime}$ untranslated region (UTR), and one mutation was in a $5^{\prime}$ UTR. PCR and conventional sequencing determined that one of the coding mutations and both UTR mutations were present both in the mutant strain and in the original, unmutagenized strain, but that the remaining coding mutation was present in the ky777 mutant but not in the original strain. This mutation represents a $\mathrm{C} \rightarrow \mathrm{T}$ transition in the predicted gene F54A5.1 that results in a missense $\mathrm{H} \rightarrow \mathrm{Y}$ mutation at the C-terminal end of the protein (Fig. 3B, red box), and was considered the most likely candidate for ky777. Transgenic introduction of a wild-type genomic copy of F54A5.1 partially restored srs $x-3$ expression in ky 777 (Fig. $4 \mathrm{C}$; see below), supporting further analysis of this gene.

F54A5.1 encodes a predicted conserved homeodomain transcription factor that contains an HNF-1 N-terminallike domain and a serine-rich region at its $\mathrm{C}$ terminus; it is closely related to the mammalian gene HMBOX1 (Fig. 3C). The homeodomain of F54A5.1 and its homologs includes an unusual 17-amino-acid insertion not present in other homeodomains (Fig. 3B, black box); the entire protein is highly conserved in multiple species, including zebrafish, mice, and humans (Fig. 3C). Because of this high degree of conservation with HMBOX1 genes, we named F54A5.1 hmbx-1.

A transgene in which GFP was expressed under the control of $7 \mathrm{~kb}$ of the $h m b x-1$ upstream region drove expression in both AWCs; in the chemosensory neurons ASI, AFD, ASH, and URX; in the mechanosensory neurons ALM, PLM, PVD, and FLP; in a few additional head and tail neurons; and in the seam cells of the hypodermis (Fig. 3D; data not shown). The expression of $h m b x-1$ in the AWCs supports the hypothesis that it regulates srsx-3 expression in these neurons. An HMBX-1 cDNA tagged with GFP localized to the nucleus of AWC, consistent with its predicted function as a transcription factor (Fig. 3E). A GFP-tagged HMBX-1(H404Y) protein corresponding to the ky777 missense mutant also localized to the nucleus.

ky777 is an altered-function allele of hmbx-1

A deletion allele of $h m b x-1, t m 1274$, was kindly provided by the National BioResource Project in Japan. The 
A

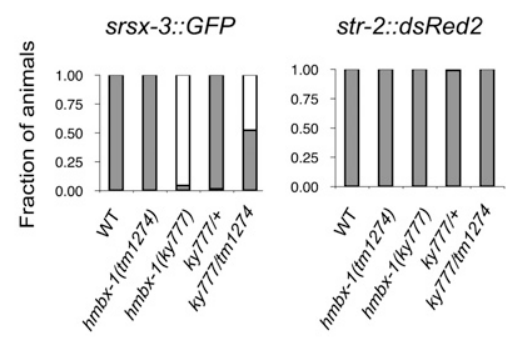

C

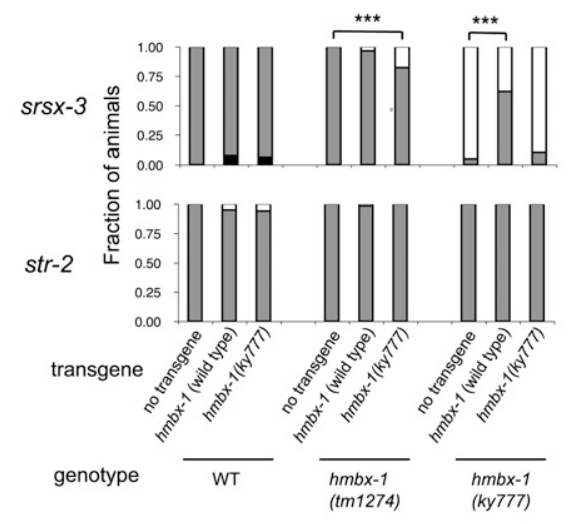

B

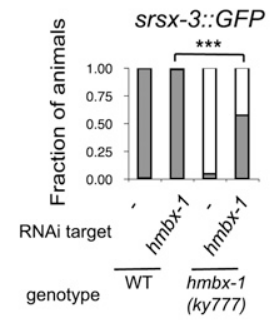

str-2::dsRed2

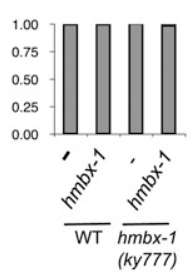

D

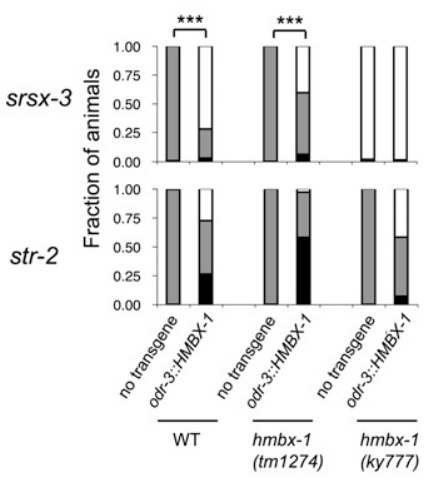

Figure 4. $\quad k y 777$ is an altered-function allele of hmbx-1. (A) srsx-3 and str-2 expression phenotypes of wild-type, hmbx-1(tm1274), and hmbx-1(ky777) homozygotes, and ky777/+ and ky777/tm1274 heterozygotes. $n>50$ for all genotypes. (B) RNAi against $h m b x-1$ in wild-type and ky777 backgrounds. All strains contained the RNAisensitizing eri-1(mg366) and 1 in-15B(n744) mutations. $\left(^{\star \star \star}\right)$ Different at $P<0.001$ (Fisher's exact test). $n>40$ for all conditions. (C) Moderate overexpression of $h m b x-1$ under endogenous regulatory elements. Expression of str-2 and srsx-3 in wild-type, ky777, or tm1274 mutant animals expressing a genomic fragment covering wild-type or ky777 mutant genomic coding sequence with $7 \mathrm{~kb}$ of upstream sequence. $\left(^{\star \star \star}\right)$ Different from transgene-negative control at $P<0.001\left(\chi^{2}\right.$ test). $n>30$ for all conditions. (D) Overexpression of $h m b x-1$ from a strong AWC promoter. Expression of str-2 and srsx-3 in wild-type, hmbx-1(tm1274), and hmbx1(ky777) animals overexpressing a wild-type HMBX-1 cDNA under the odr-3 promoter. $\left({ }^{\star \star \star}\right)$ Different from transgene-negative control at $P<0.001\left(\chi^{2}\right.$ test $) . n>60$ for all genotypes. deletion eliminates the HMBX-1 homeodomain, and results in a frameshift and early stop codon, and is therefore likely to be a null allele. The hmbx-1(tm1274) mutant was healthy and fertile, and had mild defects in chemotaxis to odors sensed by AWC neurons (Supplemental Fig. S2). Surprisingly, srsx-3 expression was normal in the hmbx-1(tm1274) mutants (Fig. 4A). The different phenotypes of the hmbx-1(ky777) and hmbx-1(tm1274) mutants indicate that ky777 is not a null allele of $h m b x-1$.

A set of genetic experiments suggested that the ky777 allele results in altered, dosage-sensitive activity of the $h m b x-1$ gene. hmbx-1(ky777)/+ animals were largely normal, indicating that ky777 is recessive to the wildtype allele (Fig. 4A). hmbx-1(ky777)/hmbx-1(tm1274null) animals had an intermediate phenotype compared with either starting strain, a result suggesting that $t m 1274$ eliminates the wild-type gene activity that suppresses $k y 777$, and supporting the hypothesis that the two mutations affect the same gene (Fig. 4A). Reducing hmbx-1 expression using RNAi in wild-type animals had little effect on srsx-3 expression, but RNAi against $h m b x-1$ in hmbx-1(ky777) mutants restored srsx-3 expression to many animals (Fig. 4B). These results suggest that RNAi is reducing an altered $h m b x-1$ activity to generate an hmbx-1-null (wild-type-like) phenotype.

A moderate increase in $h m b x-1$ activity was attained by injecting wild-type $h m b x-1$ and $h m b x-1$ (ky777) genomic DNAs into wild-type, $h m b x-1(k y 777)$, and $h m b x-$ 1(tm1274) animals. In a wild-type background, neither the wild-type nor the mutant $h m b x-1$ gene had a significant effect on srsx-3 expression, in agreement with the observation that $h m b x-1(k y 777)$ is a recessive allele (Fig. 4C). In a null background, expression of the hmbx1(ky777) mutant DNA, but not wild-type $h m b x-1$, partly repressed srsx-3 expression, confirming that hmbx1(ky777) represses srsx-3 under conditions in which the wild-type $h m b x-1$ gene does not. Finally, in a hmbx1(ky777) mutant background, wild-type $h m b x-1$ partly restored $\operatorname{srs} x-3$ expression, indicating that it antagonized hmbx-1(ky777) (Fig. 4C).

The results described above indicate that wild-type $h m b x-1$ antagonizes $h m b x-1(k y 777)$, and are consistent with two additional possibilities: (1) On its own, the $h m b x-1(k y 777)$ allele has a high or unregulated level of $h m b x-1$ activity, or (2) the $h m b x-1(k y 777)$ allele has an abnormal activity unrelated to normal $h m b x-1$ function. To distinguish between these alternatives, wild-type $h m b x-1$ cDNA was expressed at high levels in the AWC neurons using the odr-3 promoter, and the effects were examined in wild-type, $h m b x-1(t m 1274)$, and $h m b x-$ 1(ky777) animals. High-copy odr-3::hmbx-1 transgenes repressed srsx-3 in wild-type and tm1274 backgrounds, like the recessive ky777 mutant (Fig. 4D). These results suggest that $h m b x-1(k y 777)$ mutants resemble animals with increased $h m b x-1$ repressor activity in AWC. However, a few complications suggest that the effects of 
$h m b x-1$ overexpression may not be entirely straightforward. First, str-2 expression was sometimes misregulated in odr-3::hmbx-1 animals, but not in $h m b x-1$ (ky777) animals (Fig. 4D). Second, expression of hmbx-1 under the osm-3 promoter, which drives expression in 26 chemosensory neurons but not in AWC (Tabish et al. 1995), resulted in ectopic expression of srsx-3 in $\mathrm{AWC}^{\mathrm{ON}}$ in some animals (Supplemental Fig. S3). $h m b x-1$ may therefore have cell-nonautonomous as well as cell-autonomous effects on srsx-3.

Bearing these potential complications in mind, the genetic results suggest that $h m b x-1(k y 777)$ has unregulated or increased $h m b x-1$ activity that inappropriately represses $s r s x-3$ activity in AWC ${ }^{\mathrm{OFF}}$. A GFP-tagged HMBX-1(ky777) protein was expressed at similar levels to a tagged wild-type protein, suggesting that there were no major effects on protein stability. It is possible that the mutation affects an autoregulatory activity of $h m b x-1$, as expression of an $h m b x-1:: G F P$ transcriptional reporter was reduced in the AWC neurons of $h m b x-1(k y 777)$ mutants (Supplemental Fig. S3).

\section{Single-copy srsx-3::GFP transgenes are regulated}

by hmbx-1 and its predicted binding site

During the gene dosage studies, we were struck by the variation in the mutant phenotype, depending on whether the copy number of $h m b x-1$ was low (endogenous $h m b x-1)$, intermediate (genomic $h m b x-1$ DNA injection), or high (odr-3::hmbx-1 cDNA injection). In all of these experiments, str-2::dsRed2 and srsx-3::GFP reporter genes were present at high-copy number in the kyIs408 transgene, which could distort their interactions with a dosage-sensitive $h m b x-1$ transcription factor. Therefore, to provide a more natural context for examining gene regulation effects, we generated single-copy srsx-3::GFP transgene reporters at a defined site on chromosome II using the Mos Single-Copy Insertion (MosSCI) technique (Frokjaer-Jensen et al. 2008).

In wild-type animals, the single-copy srsx-3::GFP reporter recapitulated the expression pattern of a high-copy srsx-3::GFP array, albeit with a weaker GFP signal (Fig. 5A). Young larvae expressed GFP in both AWC neurons, but expression was restricted to a single $\mathrm{AWC}^{\mathrm{OFF}}$ neuron in adults (Fig. 5A; data not shown). Expression of the single-copy srsx-3::GFP transgene was lost in $h m b x-$ 1(ky777) mutants, and genetic interactions with $h m b x-$ 1(ky777) mutations were similar to those with a high-copy srsx-3::GFP array (Fig. 5A,B; Supplemental Fig. S4). Remarkably, the single-copy srs $x-3:: G F P$ reporter uncovered an opposite phenotype for the $h m b x-1(t m 1274)$-null allele: The srsx-3::GFP reporter was misexpressed in both AWCs in a fraction of $h m b x-1(t m 1274)$ adults (Fig. 5A,B). Thus, in an $h m b x-1$-null mutant, srsx-3 is derepressed in $\mathrm{AWC}^{\mathrm{ON}}$, whereas, in the $h m b x-1$ (ky777) mutant, srsx-3 is inappropriately repressed in AWC ${ }^{\mathrm{OFF}}$. These straightforward results with single-copy transgenes support and extend the conclusions from high-copy arrays. They suggest that $h m b x-1$ represses srsx-3 expression in $\mathrm{AWC}^{\mathrm{ON}}$ neurons in the adult stage, that its effect is
A

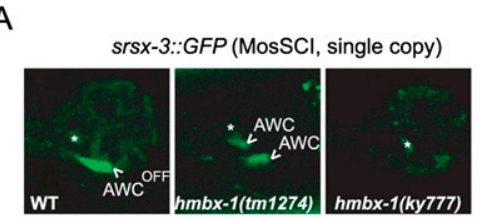

B
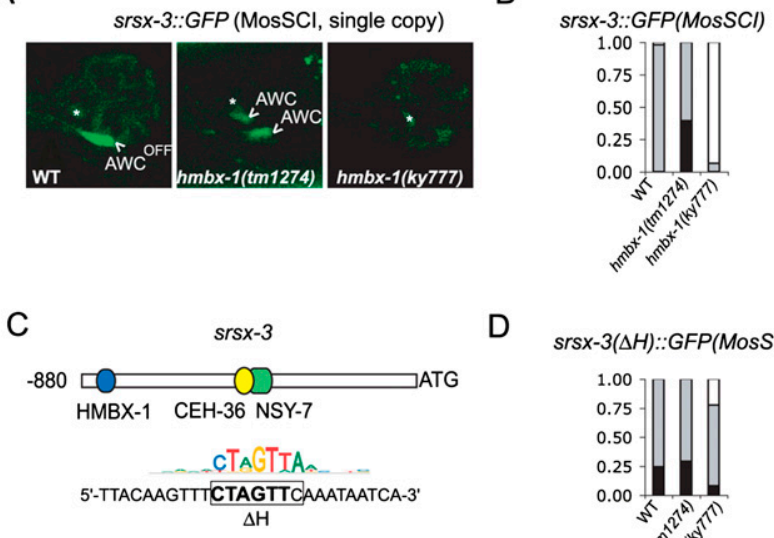

srsx-3(AH)::GFP(MosSCl)

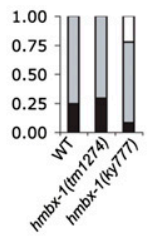

E

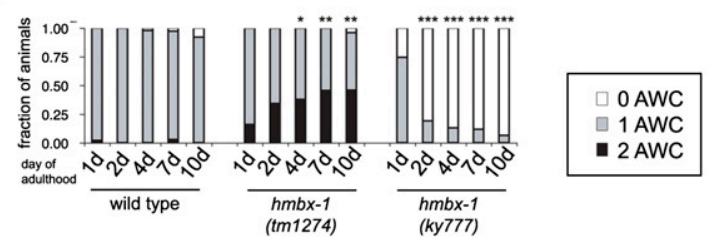

Figure 5. Regulation of single-copy srsx-3::GFP lines by $h m b x-1$. (A) Confocal images of the single-copy srsx-3::GFP reporter in wild-type, hmbx-1(tm1274), and hmbx-1(ky777) animals. (Arrowheads) AWC; (asterisks) AWB. (B) Expression of singly integrated srsx-3::GFP in wild-type and mutant adults. $n>40$ for all conditions. $(C$, top) A diagram of the srsx-3 promoter with the positions of predicted transcription factor-binding sites. (Bottom) Binding site for HMBOX1, the mouse homolog of HMBX-1, shown above the sequence in the srsx-3 promoter that was deleted in the srs $x-3(\Delta H):: G F P$ reporter. $(D)$ Phenotypes of wild-type, hmbx-1(tm1274), and hmbx-1(ky777) animals expressing the singly integrated $\operatorname{srs} x-3(\Delta H):: G F P$ reporter. $n>40$ for all genotypes. (E) Expression of singly integrated srsx$3:: G F P$ in young (1-d-old; $12 \mathrm{~h}$ after the L4 stage) and older (2-, 4-, 7-, or 10-d-old) adults in wild-type and mutant backgrounds. For hmbx-1(tm1274), one asterisk $\left(^{\star}\right)$ indicates difference from young (1-d-old) adults at $P<0.05$ and two asteriaks $\left({ }^{\star \star}\right)$ indicate difference from young adults at $P<0.01$ (Fisher's exact test). For hmbx-1(ky777), three asterisks $\left(^{\star \star \star}\right)$ indicate difference from young adults at $P<0.001$.

partly redundant with other repressors, as it is only partially penetrant, and that $h m b x-1(k y 777)$ is a recessive gain-of-function allele of $h m b x-1$ that inappropriately represses srsx-3 in AWC ${ }^{\mathrm{OFF}}$ neurons.

The binding site of the mouse homolog of HMBX-1, HMBOX1, has been identified using an in vitro binding assay (Berger et al. 2008). A similar site is present in the srsX-3 promoter, suggesting a potential site for regulation by HMBX-1 (Fig. 5C). The significance of this site was examined by deleting it from the srs -3 promoter [srsX$3(\Delta H)]$ and introducing a single-copy insertion of the srs $x$ $3(\Delta H):: G F P$ sequence into the same MosSCI site used for the wild-type srsx-3::GFP reporter. Unlike the wild-type reporter, the mutated reporter was expressed in both AWCs in a fraction of wild-type animals, suggesting that 
the sequence normally represses srsx-3 in $\mathrm{AWC}^{\mathrm{ON}}$ (Fig. $5 \mathrm{D})$. The expression of single-copy srsx-3 reporters with and without the predicted HMBX-1-binding site was then compared in wild-type, $h m b x-1(t m 1274)$, and $h m b x-$ 1(ky777) backgrounds. The single-copy srsx-3 reporter lacking the binding site behaved identically in wild-type and $h m b x-1(t m 1274)$ mutants (Fig. 5D), as predicted if HMBX-1 regulates srsx-3 expression by binding to this site. Deletion of the predicted binding site partly suppressed the effects of the hmbx-1(ky 777) mutation, suggesting that the altered-function protein also interacts with this site (Fig. $5 D$ ). Thus, the use of single-copy srsx-3::GFP reporters identified a loss-of-function phenotype for $h m b x-1$, and a likely site for HMBX-1 binding and regulation in vivo.

In agreement with the idea that $h m b x-1$ is involved in long-term maintenance of AWC gene expression, the effects of both hmbx-1(tm1274) and hmbx-1(ky777) mutants were greater in older adults than in young adults (Fig. 5E). For example, only $16 \%$ of young $h m b x-$ 1(tm1274) adults (12 h after the L4 stage) expressed srsx-3::GFP in both AWC neurons, but this fraction reached $\sim 45 \%$ by 1 wk of adulthood.

\section{Multiple repressors interact to regulate asymmetric AWC-specific genes}

Because str-2 is the only known $\mathrm{AWC}^{\mathrm{ON}}$-specific gene and srsx-3 is the only known AWC ${ }^{\mathrm{OFF}}$-specific gene, it was not clear whether transcription factors such as hmbx-1, nsy-7, and daf-3 regulate individual receptor genes or the entire asymmetric AWC identity. Therefore, we sought and characterized additional markers that distinguished the $\mathrm{AWC}^{\mathrm{ON}}$ and $\mathrm{AWC} \mathrm{CFF}^{\mathrm{OFF}}$ neurons. The C. elegans gene expression project at the University of British Columbia has reported expression of several dozen predicted chemoreceptor genes in head sensory neurons (Dupuy et al. 2007); by examining 20 of these strains, we found that the srt-26 and srt-28 reporter genes were expressed strongly in a single AWC neuron, and the srt-29 gene was expressed weakly in a single AWC. These three genes were coexpressed with str-2 but not srsx-3, indicating that their expression was specific to $\mathrm{AWC}^{\mathrm{ON}}$.

$\mathrm{AWC}^{\mathrm{OFF}}$-specific reporters were sought using the binding site for the transcription factor NSY-7, which was defined previously by direct DNA-binding experiments (Lesch et al. 2009). Fourteen predicted chemoreceptor genes in the C. elegans genome contain the CCTTAAC NSY-7-binding sequence within 300 base pairs (bp) of the coding start site. Fluorescent reporters for these 14 genes were generated by fusing $2 \mathrm{~kb}$ upstream of the start site to GFP; two of these 14 genes, srt-45 and srt-47, were expressed strongly in a single AWC neuron and weakly in an additional pair of head neurons. In both cases, expression was present in $\mathrm{AWC}^{\mathrm{OFF}}$ but not $\mathrm{AWC}^{\mathrm{ON}}$, based on coexpression with the srsx-3::mCherry reporter (Fig. 6A). It is interesting that five genes of the srt family are expressed in AWC neurons, but four other tested srt genes were expressed in different neurons, so it is not a universal pattern (data not shown).

The newly identified AWC ${ }^{\mathrm{OFF}}$ markers srt-45 and srt- 47 and the $\mathrm{AWC}^{\mathrm{ON}}$ markers srt-26 and srt-28 were examined in mutants that affect srsx-3 and str-2 expression. In nsy-7
A

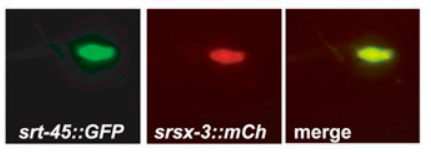

B
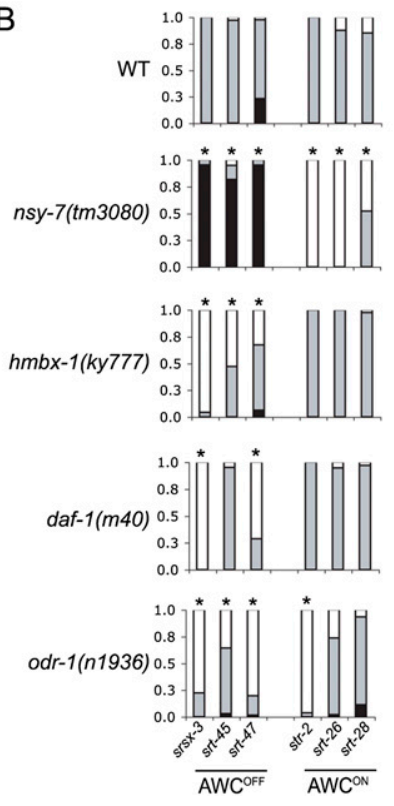
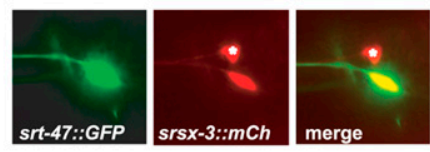

C
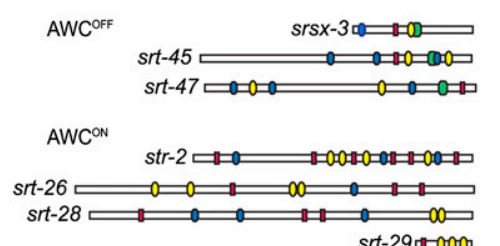

sit-29ם400000

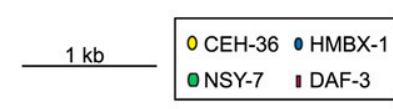

D
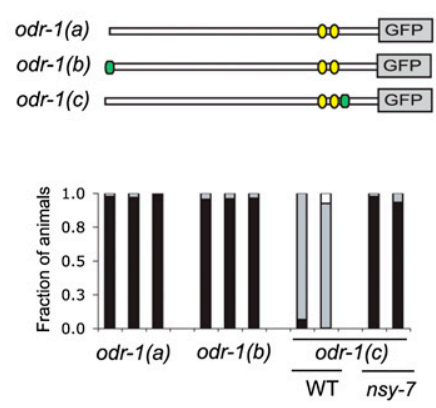

Figure 6. The NSY-7-binding site predicts $\mathrm{AWC}^{\mathrm{OFF}}$. specific expression, and is sufficient for repression in AWC $^{\mathrm{ON}}$. (A) srt-45 and srt-47 are coexpressed with the $\mathrm{AWC}^{\mathrm{OFF}}$ reporter srsx-3::mCherry, which also labels AWB (asterisks). (B) Expression of the AWC OFF markers $\left(s r s x-3, s r t-45\right.$, and srt-47) and the $\mathrm{AWC}^{\mathrm{ON}}$ markers (str-2, srt-26, and srt-28) in wild-type and mutant backgrounds. $n>30$ for all conditions; $\left(^{*}\right)$ difference from wild-type at $P<0.01$ (Fisher's exact test). (C) Schematic of $\mathrm{AWC}^{\mathrm{ON}}$ and $\mathrm{AWC}^{\mathrm{OFF}}$ promoters showing predicted binding sites for the transcription factors CEH-36, HMBX-1, NSY-7, and DAF-3. $(D$, top $)$ Schematic of odr-1::GFP constructs inserted as single copies. [odr-1(a)] Wild-type 2.4-kb odr-1 promoter fragment; [odr-1(b)] NSY-7-binding site added to the $5^{\prime}$ end of the odr-1 promoter; [odr1(c)] NSY-7-binding site added $200 \mathrm{bp}$ upstream of the ATG in the odr-1 promoter. For odr-1(c), the NSY-7-binding site is inserted close to, but does not disrupt, two CEH-36-binding sites involved in driving odr-1 expression (Kim et al. 2010). (Bottom) Expression of integrated single-copy odr-1 plasmids in wild-type and nsy-7(tm3080) backgrounds. Multiple bars within the same condition represent independently integrated lines. $n>25$ for all genotypes. 
mutants, all three $\mathrm{AWC}^{\mathrm{OFF}}$ markers were expressed in both AWCs, and all three $\mathrm{AWC}^{\mathrm{ON}}$ markers were reduced or absent (Fig. 6B). As predicted by earlier studies, this result suggests that, in nsy-7 mutants, $\mathrm{AWC}^{\mathrm{ON}}$ is transformed into $\mathrm{AWC}^{\mathrm{OFF}}$.

hmbx-1(ky777) regulated AWC ${ }^{\mathrm{OFF}}$ chemoreceptor expression in a cell-wide manner, reducing expression of all three $\mathrm{AWC}^{\mathrm{OFF}}$-specific markers, but sparing the three AWC ${ }^{\mathrm{ON}}$ markers (Fig. 6B). The symmetric AWC signaling genes odr-3 and odr-1 were expressed normally in AWC ${ }^{\mathrm{OFF}}$ neurons in $h m b x-1(k y 777)$ mutants, and AWC ${ }^{\mathrm{OFF}}$ morphology appeared normal, indicating that the $\mathrm{AWC}^{\mathrm{OFF}}$ neurons lost their asymmetric identity, but did not die or degenerate (Supplemental Fig. S5; data not shown).

Both daf-1 TGF $\beta$ mutants and odr-1 cGMP olfactory transduction mutants showed a distinct, gene-specific pattern of regulation of $\mathrm{AWC}^{\mathrm{OFF}}$ and $\mathrm{AWC}^{\mathrm{ON}}$ chemoreceptors. In daf-1 mutants, expression of the $\mathrm{AWC}{ }^{\mathrm{OFF}}$ markers srsx-3 and srt-47 was reduced, but the AWC ${ }^{\mathrm{OFF}}$ marker srt-45 and all AWC ${ }^{\mathrm{ON}}$ markers were expressed at wild-type levels (Fig. 6B). odr-1 mutants were defective for expression of all three $\mathrm{AWC}^{\mathrm{OFF}}$ markers and the $\mathrm{AWC}^{\mathrm{ON}}$ marker str-2, but expression of the $\mathrm{AWC}^{\mathrm{ON}}$ markers srt-26 and srt-28 was largely intact (Fig. 6B). Thus, the TGF $\beta$ and cGMP-dependent pathways regulate subsets of chemoreceptor genes in both $\mathrm{AWC}^{\mathrm{OFF}}$ and $\mathrm{AWC}^{\mathrm{ON}}$ neurons.

The relationships between the different transcriptional pathways were probed by examining double mutants, using the multicopy str-2 and srsx-3 reporters and the srs $x-3$ single-copy reporter. In general, double mutants recapitulated single mutant phenotypes in simple patterns, suggesting that $h m b x-1, n s y-7$, and daf-3 act independently of each other (Supplemental Fig. S6). odr-1 defects were partly suppressed by hmbx-1(tm1274), suggesting that these two pathways are additive and independent; $h m b x-1(k y 777)$ and nsy-7 effects were also additive.

The seven asymmetric AWC promoters had many potential binding sites for AWC-regulating proteins (Fig. 6C). All seven promoter fragments had predicted binding sites for CEH-36, the AWC terminal selector gene, and potential binding sites for DAF-3; six of seven promoters had matches to the consensus binding site for HMBX-1. NSY-7 sites were only present in the three AWC ${ }^{\mathrm{OFF}}$ promoters. In previous studies, analysis of the srs $x-3$ promoter suggested that NSY-7 binding was necessary for asymmetric expression (Lesch et al. 2009). The identification of additional transcriptional repressors raised the question of whether NSY-7 binding was also sufficient for asymmetric expression. To address this question, a single copy of the CCTTAAC sequence was inserted into an odr-1::GFP reporter, which is ordinarily expressed in both AWC neurons (and also in AWB neurons). Single-copy odr-1::GFP reporters with and without CCTTAAC sequences were inserted at a defined site on chromosome II using the MosSCI method. The wild-type single-copy odr-1::GFP fusion was expressed in both AWC neurons, but an odr-1 plasmid with a CCTTAAC site $200 \mathrm{bp}$ upstream of the ATG was expressed asymmetrically in a single AWC neuron (Fig. 6D). Coexpression of this transgene with srs $x-3$ but not str-2 reporters indicated that the CCTTAAC site repressed expression in $\mathrm{AWC}^{\mathrm{ON}}$. When crossed into a nsy-7 mutant, the modified transgene was again expressed in both AWC neurons (Fig. 6D). A more distal insertion of the NSY-7 site was expressed bilaterally. These results support the hypothesis that NSY-7 is a transcriptional repressor, and demonstrate that a single promoterproximal NSY-7-binding site is sufficient to repress gene expression in $\mathrm{AWC}^{\mathrm{ON}}$.

\section{Discussion}

Multiple environmental and cell-intrinsic influences converge at the transcriptional level to regulate chemoreceptors in AWC neurons. Together with the symmetric AWC terminal selector gene ceh-36 (Kim et al. 2010), at least four different systems for transcriptional regulation contribute to adult $\mathrm{AWC}^{\mathrm{OFF}}$-specific expression of the chemoreceptor gene srsx-3. First, the previously identified transcriptional repressor NSY-7, which is expressed preferentially in $\mathrm{AWC}^{\mathrm{ON}}$, can repress srsx-3 expression by direct binding to a consensus site. Second, cGMP signaling promotes srs $x-3$ expression via a cGMP-dependent protein kinase, a cell-autonomous cGMP-gated channel, and unknown transcriptional regulators (Lesch et al. 2009). Third, density-dependent dauer pheromones repress expression of the secreted TGF $\beta$ homolog DAF-7, which otherwise acts continuously to maintain srs $x-3$ expression. The TGF $\beta$ pathway regulates multiple chemoreceptor genes; in agreement with this observation, binding sites for SMAD transcription factors such as DAF-3 are among the most common sequences found upstream of chemoreceptor start sites (McCarroll et al. 2005). Finally, the conserved transcription factor $h m b x-1$ represses srs $x-3$ expression preferentially in adult $\mathrm{AWC}^{\mathrm{ON}}$ neurons, although it is expressed in both AWC neurons and can act in $\mathrm{AWC}^{\mathrm{OFF}}$ when bearing the ky777 point mutation or when overexpressed.

Genetic analysis of $h m b x-1$ suggests that it is involved in long-term maintenance of a specific cell identity, not the developmental establishment of that identity or the regulation of specific genes. The original hmbx-1(ky777) mutation is a recessive gain-of-function allele with effects that resemble those of $h m b x-1$ overexpression. Because of its nature and its dosage sensitivity, it was identified only by whole-genome sequencing. Although recessive gain-of-function alleles are relatively rare, they have been described in genes encoding potassium channels and their regulators, tyrosine kinase receptors, and the ETS domain transcription factor LIN-1 (Klingler et al. 1988; Jacobs et al. 1998; Perez de la Cruz et al. 2003). LIN1-recessive gain-of-function mutations disrupt a negative regulatory domain; it is possible that ky777 does the same to HMBX-1.

The analysis of a single-copy insertion of the srsx$3:: G F P$ reporter uncovered a phenotype for the $h m b x-$ 1-null mutant that was not evident with a multicopy reporter, demonstrating that $h m b x-1$ normally represses 
$\mathrm{AWC}^{\mathrm{OFF}}$ chemoreceptor genes in $\mathrm{AWC}^{\mathrm{ON}}$ neurons. Singlecopy reporter genes also identified a predicted HMBX1-binding site required for repression of srsx-3 by hmbx-1. Multicopy integrated and extrachromosomal arrays are commonly and successfully used to analyze gene expression in C. elegans. However, high-copy transgenes are subject to repeat-induced silencing in the germline and, to a lesser extent, somatic tissues (Hsieh and Fire 2000), and this leads to altered genetic requirements for their expression. For example, specific genes including tam-1, a gene isolated in our screen, are required for efficient expression of high-copy transgenes, but not of the corresponding endogenous genes (Hsieh et al. 1999). Moreover, high-copy transgenes have a high propensity to form heterochromatin, which is not observed with low-copy transgenes of the same sequence (Meister et al. 2010). We suggest that the heterochromatic state of the high-copy transgene may bypass the normal requirement for $h m b x-1$ and perhaps other repressors that maintain gene silencing. The ability to introduce single-copy transgenes into defined genomic locations by MosSCI represents a significant advance for controlling copy number and genomic context effects on gene expression (Frokjaer-Jensen et al. 2008).

$h m b x-1$ has effects that are temporally distinct from those of $n s y-7$, a transcription factor that acts in $\mathrm{AWC}^{\mathrm{ON}}$ beginning in the $\mathrm{L} 1$ larval stage. The relative importance of wild-type $h m b x-1$ is greater in older compared with younger adults, and, likewise, the elevated repressive activity of the hmbx-1(ky777) mutant increases in adults over time. It may be that NSY-7 loses activity in older animals, or that other changes in gene expression alter the relative importance of the two transcription factors as the animal ages.

The results described here point to a significant role for single transcription factor-binding sites in chemoreceptor expression. The presence of a single binding site for NSY-7 is sufficient to repress expression of an odr-1 promoter ordinarily expressed in both AWC neurons. Likewise, deletion or mutation of the HMBX-1-binding site in the $s r s x-3$ promoter permitted misexpression of srs $x-3$ in AWC ${ }^{\mathrm{ON}}$. The relative ease with which sensory receptor expression can be altered suggests genetic malleability and potentially evolutionary flexibility in the specificity of GPCR expression in chemosensory neurons (Jovelin 2009). Particularly in C. elegans, where ectopic expression of a gene in a single additional neuron can dramatically change behaviors, altered expression due to new mutations might have significant consequences for the animal's behavior and fitness (Troemel et al. 1997).

Despite the potent effects of single binding sites, many additional candidate binding sites were identified in each asymmetric AWC gene. Functional studies of asymmetrically expressed promoters in ASE neurons have uncovered multiple repressor and activator elements in each promoter (Etchberger et al. 2009). In ASE, che-1-binding sites with different affinities contribute to left-right asymmetry of gene expression as well as general ASE identity (Etchberger et al. 2009); it may be informative that the $\mathrm{AWC}^{\mathrm{ON}}$-specific promoters contain more pre- dicted $\mathrm{CEH}$-36-binding sites than the $\mathrm{AWC}^{\mathrm{OFF}}$-specific promoters.

$h m b x-1$ is expressed in C. elegans sensory neurons, and its human and mouse homologs also display high levels of nervous system expression (Chen et al. 2006). The human homolog exhibits repressor activity in vitro, and associates with telomeric sequences in several human cell lines (Chen et al. 2006; Dejardin and Kingston 2009). Telomeres are zones of strong transcriptional repression, suggesting a possible repressor function for HMBOX-1 at these locations (Gottschling et al. 1990). The sequence conservation of HMBOX-1 genes between worm and mammalian homologs, their conserved repressor activity, the apparently conserved binding site specificity, and their neuronal expression pattern all hint at possible conserved neuronal functions. With this in mind, it will be interesting to determine the identities of $h m b x-1$ target genes, particularly those that are important in older adult animals, and to explore the association of human HMBOX1 with telomeric repeats during normal growth and aging.

\section{Materials and methods}

\section{Genetics and strains}

C. elegans strains were cultured using standard methods (Brenner 1974). All strains were grown at $20^{\circ} \mathrm{C}$ unless otherwise specified. Mutants were isolated by direct inspection of GFP and dsRed fluorescence following mutagenesis of the strain CX7894 kyIs408 [srsx-3::GFP;str-2::dsRed2;elt-2::GFP] II (Lesch et al. 2009). Mutagenesis was performed with ethyl methane sulfonate (EMS) using standard protocols (Brenner 1974).

Among the new mutations, tax-4 and tax-2 alleles differed in phenotype from tax-4 and tax-2 mutants characterized previously using the kyIs140 [str-2::GFP] I transgene (Troemel et al. 1999; Lans and Jansen 2006). Whereas expression of str-2::GFP from kyIs140 was lost in most tax-2 and tax-4 mutants, the effect of tax-2 and tax-4 on str-2::DsRed from kyIs 408 was milder. Analysis of the canonical tax-4(p678)-null mutant indicated that the difference was due to the transgene and not to the nature of the mutant alleles (Fig. 1B). kyIs140 fluorescence is much dimmer than that of kyIs 408 , so it may be more sensitive to small reductions in expression level.

A strain list appears as Supplemental Table S1.

\section{Molecular biology}

Standard molecular biology techniques were used. The gfp-pest reporter was made by overlap extension PCR between the GFP template from the pSM-GFP expression vector and the PEST domain template from a commercially available destabilized GFP vector (d1EGFP-N1, Clontech). The product was then cloned into MCS2 of pSM with the srsx-3 promoter in MCS1 to create the srsx-3::gfp-pest vector.

A PCR fragment containing the $h m b x-1$ genomic coding region and $7 \mathrm{~kb}$ of upstream sequence was amplified from N2 lysate using the primers 5'-CATATCACATCCTCCTGTTTTC AAG-3' and 5' -CCAACAAAATTTCAGGGCAAGC-3' . HMBX-1 cDNAs were obtained by PCR from a C. elegans poly(A) cDNA library using primers at the beginning and end of the predicted ORF (5'-ATGCTATTCACAATTGAGCAACTG- ${ }^{\prime}$ ' and $5^{\prime}$-TCA CTCAGACTTCAAAATAGAAG-3'), and were cloned into 
MCS2 of a modified pSM expression vector (containing a NotI site in MCS2 instead of MCS1) using the restriction sites Sall and NotI.

The $h m b x-1(7 k b):: G F P$, srt-45::GFP, and srt-47::GFP reporters were created by PCR fusion of the promoter, amplified from N2 lysate, with GFP template amplified from pPD95.75. Reactions were carried out as described in Hobert (2002) with the following primers: $h m b x-1(7 k b)$ : A, $5^{\prime}$-TGCCGATTGCCAAAA ACTTCC-3', and A $^{*}, 5^{\prime}$-CATATCACATCCTCCTGTTTTCA AG-3', and B, 5' -AGTCGACCTGCAGGCATGCAAGCTGTGT AAAATAAAATTTACTACGAGGTT-3'; srt-45: A, 5' -CTTTTA AGTATTGTCACTCCGGC-3' , and A $^{*}, 5^{\prime}$-TTGGACATGTAAT CATGCATG-3' ${ }^{\prime}$, and B, $5^{\prime}$-AGTCGACCTGCAGGCATGCAAG CTTGCGGAATCTGAAGTTTTTCG-3'; srt-47: A, 5'-GCGTA AAACACAGCAGAAAA- ${ }^{\prime}$, and $\mathrm{A}^{*}{ }^{*}, 5^{\prime}$-ATCTTTAAAGGTGC ATTTTATTTGG-3', and B, 5' -AGTCGACCTGCAGGCATGC AAGCTTTGGTGATAAAGAGAGGTTATAG-3' .

To create the srt-26::GFP, srt-28::GFP, and srt-29::GFP reporters, the srt-26, srt-28, and srt-29 promoter sequences were amplified by PCR and cloned into MCS1 of the pSM-GFP expression vector using the FseI and AscI restriction sites.

For MosSCI experiments, NSY-7-binding sites were added to the odr-1::GFP expression vector, and the srsx-3::GFP plasmid was modified to generate the $s r s x-3(\Delta H):: G F P$ reporter by sitedirected mutagenesis using the the QuikChange II Site-Directed Mutagenesis Kit (Stratagene). odr-1::GFP or srsx-3::GFP sequences were cut out of pSM using the FseI and SpeI restriction sites, and were cloned into a pCFJ151 MosSCI insertion vector (Frokjaer-Jensen et al. 2008) that had been modified to include a FseI site in the MCS.

\section{Whole-genome sequencing}

Whole-genome sequencing of the ky777 mutant was performed by the Rockefeller Genomics Resource Center using SolexaIllumina Genome Analyzer technology. SNP mapping was used to determine a genetic interval of $\sim 440 \mathrm{~kb}$ for the mutation. Purified genomic DNA was prepared from 1000-2000 worms, and was used for library preparation. fastq sequences were aligned to the ce6 genome assembly at the University of California at Santa Cruz using the Mosaik alignment program (http://bioinformatics.bc.edu/marthlab/Mosaik\#Current_ Documentation), and single-base changes, insertions, or deletions were predicted using GigaBayes polymorphism detection software (http://bioinformatics.bc.edu/marthlab/GigaBayes). High-probability single-base changes that were predicted to fall within an exon, a 3'UTR, or a 5'UTR were checked by PCR and conventional (Sanger) sequencing.

\section{Sequence alignment}

The hmbx-1 homeodomain sequence was aligned with HMBOX1 homeodomain sequences using ClustalW at Pôle BioInformatique Lyonnais (http://npsa-pbil.ibcp.fr/cgi-bin/npsa automat.pl?page=npsa_clustalw.html). UniProt ID numbers are as follows: for C. elegans, Q9TYT0; for Brugia malayi, A8QCW9; for Danio rerio, Q4V904; for Mus musculus, Q8BJA3; for Homo sapiens, A8K3R8.

\section{RNAi}

RNAi was performed by injection of dsRNA. All assays were performed in an eri-1(mg366); 1 in-15B(n744)-sensitized background (Sieburth et al. 2005). For injection, a dsDNA template corresponding to an exon of the target ORF was amplified from N2 lysate with a T7 sequence (TAATACGACTCACTATAGGG AGA) added at the $5^{\prime}$ ends. The following gene-specific sequences were used for these primers: nsy-7 (exon 2), 5' -GTTGCG AAAGGATATTCAGATG- ${ }^{\prime}$ ' and $5^{\prime}$-CTTAGCAAACAAGTTG GTGAGT-3'; $h m b x-1$ (exon 3), 5'-ACAAGCTCCCGTAACAC AAC-3' and 5' -TCACTCAGACTTCAAAATAGAAGCC-3'.

Transcription was performed using the T7 RiboMAX Express RNAi System (Promega) according to instructions, and the unpurified reaction mix was injected into the body cavity, gut, or gonad of adult hermaphrodites. F1 progeny from eggs laid at least $24 \mathrm{~h}$ after the injection were scored after 3-4 $\mathrm{d}$ for str-2 and srsx-3 expression. Control experiments established the normal RNAi response of $h m b x-1$ (ky 777) animals: RNAi against nsy-7 in ky777 mutants resulted in a 100\% loss of str-2 expression (44 out of $44 \mathrm{RNAi}^{+}$vs. zero out of $90 \mathrm{RNAi}^{-}$animals; $P<0.0001$ [Fisher's exact test]), consistent with the phenotype of $h m b x$ 1(ky777);nsy-7(tm3080) double mutants.

\section{MosSCI integrations}

Mos single-copy integrants were generated using the direct insertion protocol described in Frokjaer-Jensen et al. (2008). Thirty to 50 EG4322 ttTi5605; unc-119(ed3) worms were injected with rab-3::mCherry, myo-2::mCherry, myo-3::mCherry, pJL43.1 (a vector containing the Mos1 transposase under the control of the germline promoter glh-2), and a vector containing the specific promoter::GFP sequence to be inserted flanked by sequences homologous to the insertion site. Animals that were rescued for the unc-119 phenotype (array-positive) were allowed to starve out twice, and then unc-119 rescued animals that lacked the three mCherry coinjection markers (integrant-positive, arraynegative) were cloned out from separate plates to found independent integrated lines. These lines were outcrossed twice to wild-type animals, and the presence of the intact insertion was verified by PCR and sequencing.

\section{Microscopy}

For all microscopy, live animals were immobilized on an agarose pad containing $5 \mathrm{mM} \mathrm{NaN}_{3}$.

Fluorescence microscopy was carried out on a Zeiss Axioplan2 imaging system with a Hamamatsu Photonics C2400 CCD camera, or a Zeiss Axio Imager.Z1 with ApoTome with a Zeiss AxioCam MRm CCD camera. Most animals were scored under a $20 \times$ or $40 \times$ Plan-Neofluar objective, where "bright" and "faint" fluorescence were scored qualitatively, and photographs were taken under a $40 \times$ Plan-Neofluar or $63 \times$ Plan-Apochromat objective. Confocal microscopy (Fig. 5A) was done under a 40×/ $1.2 \mathrm{~W}$ C-Apochromat water immersion objective on a Zeiss LSM 510 confocal imaging system using the Zeiss LSM 510 version 3.2 confocal software.

\section{Developmental timing}

To evaluate marker expression in the $\mathrm{L} 1$ larval stage, larvae were staged by hatch-off. Late embryos were picked to an NGM plate seeded with the Escherichia coli strain OP50. After $30 \mathrm{~min}$, justhatched L1s were transferred to a fresh plate and grown for $14 \mathrm{~h}$ at $20^{\circ} \mathrm{C}$ (for L1s) or $\sim 70 \mathrm{~h}$ at $20^{\circ} \mathrm{C}$ (for adults). To compare young and old adults, $25 \mathrm{~L} 4$ animals were picked per plate and grown for $12 \mathrm{~h}(1 \mathrm{~d})$ at $20^{\circ} \mathrm{C}, 36 \mathrm{~h}(2 \mathrm{~d})$ at $20^{\circ} \mathrm{C}, 84 \mathrm{~h}(4 \mathrm{~d})$ at $20^{\circ} \mathrm{C}, 156 \mathrm{~h}(7 \mathrm{~d})$ at $20^{\circ} \mathrm{C}$, or $228 \mathrm{~h}(10 \mathrm{~d})$ at $20^{\circ} \mathrm{C}$ before scoring. Animals were transferred to new plates every $24 \mathrm{~h}$ to prevent crowding and starvation. 


\section{Pheromone assays}

Ascarosides C3, C6, and C9 (generously provided by R. Butcher and J. Clardy, Harvard Medical School) were added to liquid agar at the concentrations indicated. For negative controls, the same volume of solvent (ethanol) was added to the agar. Ten milliliters of agar was poured into 6-cm culture dishes and allowed to cool. Plates were then seeded with $100 \mu \mathrm{L}$ of OP50 bacteria and dried in a hood for 1.5-2 h. These plates were either used immediately or stored overnight at $4^{\circ} \mathrm{C}$. Twenty srsx-3::gfp-pest arraypositive young adults (older than L4, with no eggs yet visible in the gonad) were picked to each plate and incubated for $4 \mathrm{~h}$ at $25^{\circ} \mathrm{C}$. Animals were scored for presence or absence of GFP in AWC neurons under a $40 \times$ objective.

\section{Acknowledgments}

We thank Shohei Mitani for the hmbx-1(tm1274) strain, Rebecca Butcher and Jon Clardy for ascaroside pheromones, and Mike Chiorazzi and Diana McKeage for contributing to the mutant characterization. This work was supported by NIDCD grant DC004089. B.J.L. was supported by MSTP grant GM07739, and by an individual NRSA (F30MH084482). C.I.B is an Investigator of the Howard Hughes Medical Institute.

\section{References}

Berger MF, Badis G, Gehrke AR, Talukder S, Philippakis AA, Pena-Castillo L, Alleyne TM, Mnaimneh S, Botvinnik OB, Chan ET, et al. 2008. Variation in homeodomain DNA binding revealed by high-resolution analysis of sequence preferences. Cell 133: 1266-1276.

Brenner S. 1974. The genetics of Caenorhabditis elegans. Genetics 77: 71-94.

Buck L, Axel R. 1991. A novel multigene family may encode odorant receptors: A molecular basis for odor recognition. Cell 65: 175-187.

Busturia A, Morata G. 1988. Ectopic expression of homeotic genes caused by the elimination of the Polycomb gene in Drosophila imaginal epidermis. Development 104: 713-720.

Butcher RA, Fujita M, Schroeder FC, Clardy J. 2007. Smallmolecule pheromones that control dauer development in Caenorhabditis elegans. Nat Chem Biol 3: 420-422.

Butcher RA, Ragains JR, Kim E, Clardy J. 2008. A potent dauer pheromone component in Caenorhabditis elegans that acts synergistically with other components. Proc Natl Acad Sci 105: 14288-14292.

Cao R, Wang L, Wang H, Xia L, Erdjument-Bromage H, Tempst P, Jones RS, Zhang Y. 2002. Role of histone H3 lysine 27 methylation in Polycomb-group silencing. Science 298: 1039-1043.

Chalasani SH, Chronis N, Tsunozaki M, Gray JM, Ramot D, Goodman MB, Bargmann CI. 2007. Dissecting a circuit for olfactory behaviour in Caenorhabditis elegans. Nature 450: 63-70.

Chang S, Johnston RJ Jr, Hobert O. 2003. A transcriptional regulatory cascade that controls left/right asymmetry in chemosensory neurons of C. elegans. Genes Dev 17: 2123-2137.

Chang S, Johnston RJ Jr, Frokjaer-Jensen C, Lockery S, Hobert O. 2004. MicroRNAs act sequentially and asymmetrically to control chemosensory laterality in the nematode. Nature 430: 785-789.

Chen S, Saiyin H, Zeng X, Xi J, Liu X, Li X, Yu L. 2006. Isolation and functional analysis of human HMBOX1, a homeobox containing protein with transcriptional repressor activity. Cytogenet Genome Res 114: 131-136.
Clyne PJ, Warr CG, Carlson JR. 2000. Candidate taste receptors in Drosophila. Science 287: 1830-1834.

Coburn CM, Mori I, Ohshima Y, Bargmann CI. 1998. A cyclic nucleotide-gated channel inhibits sensory axon outgrowth in larval and adult C. elegans: A distinct pathway for maintenance of sensory axon structure. Development 125: 249-258.

Colosimo ME, Tran S, Sengupta P. 2003. The divergent orphan nuclear receptor ODR-7 regulates olfactory neuron gene expression via multiple mechanisms in Caenorhabditis elegans. Genetics 165: 1779-1791.

da Graca LS, Zimmerman KK, Mitchell MC, Kozhan-Gorodetska M, Sekiewicz K, Morales Y, Patterson GI. 2004. DAF-5 is a Ski oncoprotein homolog that functions in a neuronal TGF $\beta$ pathway to regulate C. elegans dauer development. Development 131: 435-446.

Dejardin J, Kingston RE. 2009. Purification of proteins associated with specific genomic loci. Cell 136: 175-186.

Dupuy D, Bertin N, Hidalgo CA, Venkatesan K, Tu D, Lee D, Rosenberg J, Svrzikapa N, Blanc A, Carnec A, et al. 2007. Genome-scale analysis of in vivo spatiotemporal promoter activity in Caenorhabditis elegans. Nat Biotechnol 25: 663668.

Etchberger JF, Lorch A, Sleumer MC, Zapf R, Jones SJ, Marra MA, Holt RA, Moerman DG, Hobert O. 2007. The molecular signature and cis-regulatory architecture of a C. elegans gustatory neuron. Genes Dev 21: 1653-1674.

Etchberger JF, Flowers EB, Poole RJ, Bashllari E, Hobert O. 2009. Cis-regulatory mechanisms of left/right asymmetric neuronsubtype specification in C. elegans. Development 136: 147160.

Frand AR, Russel S, Ruvkun G. 2005. Functional genomic analysis of C. elegans molting. PLoS Biol 3: e312. doi: 10.1371/ journal.pbio.0030312.

Frokjaer-Jensen C, Davis MW, Hopkins CE, Newman BJ, Thummel JM, Olesen SP, Grunnet M, Jorgensen EM. 2008. Single-copy insertion of transgenes in Caenorhabditis elegans. Nat Genet 40: 1375-1383.

Gaudet J, Mango SE. 2002. Regulation of organogenesis by the Caenorhabditis elegans FoxA protein PHA-4. Science 295: 821-825.

Georgi LL, Albert PS, Riddle DL. 1990. daf-1, a C. elegans gene controlling dauer larva development, encodes a novel receptor protein kinase. Cell 61: 635-645.

Gottschling DE, Aparicio OM, Billington BL, Zakian VA. 1990. Position effect at $S$. cerevisiae telomeres: Reversible repression of Pol II transcription. Cell 63: 751-762.

Hobert O. 2002. PCR fusion-based approach to create reporter gene constructs for expression analysis in transgenic $C$. elegans. Biotechniques 32: 728-730.

Hobert O. 2008. Regulatory logic of neuronal diversity: Terminal selector genes and selector motifs. Proc Natl Acad Sci 105: 20067-20071.

Hsieh J, Fire A. 2000. Recognition and silencing of repeated DNA. Annu Rev Genet 34: 187-204.

Hsieh J, Liu J, Kostas SA, Chang C, Sternberg PW, Fire A. 1999. The RING finger/B-box factor TAM-1 and a retinoblastomalike protein LIN-35 modulate context-dependent gene silencing in Caenorhabditis elegans. Genes Dev 13: 29582970.

Jacobs D, Beitel GJ, Clark SG, Horvitz HR, Kornfeld K. 1998. Gain-of-function mutations in the Caenorhabditis elegans lin-1 ETS gene identify a C-terminal regulatory domain phosphorylated by ERK MAP kinase. Genetics 149: 18091822.

Jeong PY, Jung M, Yim YH, Kim H, Park M, Hong E, Lee W, Kim YH, Kim K, Paik YK. 2005. Chemical structure and 
biological activity of the Caenorhabditis elegans dauerinducing pheromone. Nature 433: 541-545.

Johnston RJ, Hobert O. 2003. A microRNA controlling left/right neuronal asymmetry in Caenorhabditis elegans. Nature 426: 845-849.

Johnston RJ Jr, Chang S, Etchberger JF, Ortiz CO, Hobert O. 2005. MicroRNAs acting in a double-negative feedback loop to control a neuronal cell fate decision. Proc Natl Acad Sci 102: $12449-12454$.

Jovelin R. 2009. Rapid sequence evolution of transcription factors controlling neuron differentiation in Caenorhabditis. Mol Biol Evol 26: 2373-2386.

Kim K, Colosimo ME, Yeung H, Sengupta P. 2005. The UNC-3 Olf/EBF protein represses alternate neuronal programs to specify chemosensory neuron identity. Dev Biol 286: 136148.

Kim K, Sato K, Shibuya M, Zeiger DM, Butcher RA, Ragains JR, Clardy J, Touhara K, Sengupta P. 2009. Two chemoreceptors mediate developmental effects of dauer pheromone in $C$. elegans. Science 326: 994-998.

Kim K, Kim R, Sengupta P. 2010. The HMX/NKX homeodomain protein MLS-2 specifies the identity of the AWC sensory neuron type via regulation of the ceh-36 Otx gene in $C$. elegans. Development 137: 963-974.

Klingler M, Erdelyi M, Szabad J, Nusslein-Volhard C. 1988. Function of torso in determining the terminal anlagen of the Drosophila embryo. Nature 335: 275-277.

Lanjuin A, Sengupta P. 2002. Regulation of chemosensory receptor expression and sensory signaling by the KIN-29 Ser/Thr kinase. Neuron 33: 369-381.

Lanjuin A, VanHoven MK, Bargmann CI, Thompson JK, Sengupta P. 2003. Otx/otd homeobox genes specify distinct sensory neuron identities in C. elegans. Dev Cell 5: 621-633.

Lans H, Jansen G. 2006. Noncell- and cell-autonomous G-protein-signaling converges with $\mathrm{Ca} 2+/$ mitogen-activated protein kinase signaling to regulate str-2 receptor gene expression in Caenorhabditis elegans. Genetics 173: 12871299.

Lesch BJ, Gehrke AR, Bulyk ML, Bargmann CI. 2009. Transcriptional regulation and stabilization of left-right neuronal identity in C. elegans. Genes Dev 23: 345-358.

McCarroll SA, Li H, Bargmann CI. 2005. Identification of transcriptional regulatory elements in chemosensory receptor genes by probabilistic segmentation. Curr Biol 15: 347-352.

Meister P, Towbin BD, Pike BL, Ponti A, Gasser SM. 2010. The spatial dynamics of tissue-specific promoters during $C$. elegans development. Genes Dev 24: 766-782.

Mikkola I, Heavey B, Horcher M, Busslinger M. 2002. Reversion of B cell commitment upon loss of Pax5 expression. Science 297: 110-113.

Muller J, Kassis JA. 2006. Polycomb response elements and targeting of Polycomb group proteins in Drosophila. Curr Opin Genet Dev 16: 476-484.

Nokes EB, Van Der Linden AM, Winslow C, Mukhopadhyay S, Ma K, Sengupta P. 2009. Cis-regulatory mechanisms of gene expression in an olfactory neuron type in Caenorhabditis elegans. Dev Dyn 238: 3080-3092.

Nolan KM, Sarafi-Reinach TR, Horne JG, Saffer AM, Sengupta P. 2002 . The DAF-7 TGF- $\beta$ signaling pathway regulates chemosensory receptor gene expression in C. elegans. Genes Dev 16: 3061-3073.

Nutt SL, Heavey B, Rolink AG, Busslinger M. 1999. Commitment to the B-lymphoid lineage depends on the transcription factor Pax5. Nature 401: 556-562.

Patterson GI, Koweek A, Wong A, Liu Y, Ruvkun G. 1997. The DAF-3 Smad protein antagonizes TGF- $\beta$-related receptor signaling in the Caenorhabditis elegans dauer pathway. Genes Dev 11: 2679-2690.

Peckol EL, Troemel ER, Bargmann CI. 2001. Sensory experience and sensory activity regulate chemosensory receptor gene expression in Caenorhabditis elegans. Proc Natl Acad Sci 98: $11032-11038$.

Perez de la Cruz I, Levin JZ, Cummins C, Anderson P, Horvitz HR. 2003. sup-9, sup-10, and unc-93 may encode components of a two-pore $\mathrm{K}^{+}$channel that coordinates muscle contraction in Caenorhabditis elegans. J Neurosci 23: 91339145 .

Prasad BC, Ye B, Zackhary R, Schrader K, Seydoux G, Reed RR. 1998. unc-3, a gene required for axonal guidance in Caenorhabditis elegans, encodes a member of the O/E family of transcription factors. Development 125: 1561-1568.

Ren P, Lim CS, Johnsen R, Albert PS, Pilgrim D, Riddle DL. 1996. Control of C. elegans larval development by neuronal expression of a TGF- $\beta$ homolog. Science 274: 1389-1391.

Rens-Domiano S, Hamm HE. 1995. Structural and functional relationships of heterotrimeric G-proteins. FASEB I 9: 10591066.

Ringrose L, Paro R. 2004. Epigenetic regulation of cellular memory by the Polycomb and Trithorax group proteins. Annu Rev Genet 38: 413-443.

Sagasti A, Hobert O, Troemel ER, Ruvkun G, Bargmann CI. 1999. Alternative olfactory neuron fates are specified by the LIM homeobox gene lim-4. Genes Dev 13: 1794-1806.

Sarafi-Reinach TR, Sengupta P. 2000. The forkhead domain gene unc-130 generates chemosensory neuron diversity in $C$. elegans. Genes Dev 14: 2472-2485.

Sarafi-Reinach TR, Melkman T, Hobert O, Sengupta P. 2001. The lin-11 LIM homeobox gene specifies olfactory and chemosensory neuron fates in C. elegans. Development 128: 3269-3281.

Schackwitz WS, Inoue T, Thomas JH. 1996. Chemosensory neurons function in parallel to mediate a pheromone response in C. elegans. Neuron 17: 719-728.

Sengupta P, Colbert HA, Bargmann CI. 1994. The C. elegans gene odr-7 encodes an olfactory-specific member of the nuclear receptor superfamily. Cell 79: 971-980.

Shibata Y, Takeshita H, Sasakawa N, Sawa H. 2010. Double bromodomain protein BET-1 and MYST HATs establish and maintain stable cell fates in C. elegans. Development 137: 1045-1053.

Shirasaki R, Pfaff SL. 2002. Transcriptional codes and the control of neuronal identity. Annu Rev Neurosci 25: 251281.

Sieburth D, Ch'ng Q, Dybbs M, Tavazoie M, Kennedy S, Wang D, Dupuy D, Rual JF, Hill DE, Vidal M, et al. 2005. Systematic analysis of genes required for synapse structure and function. Nature 436: 510-517.

Tabish M, Siddiqui ZK, Nishikawa K, Siddiqui SS. 1995. Exclusive expression of C. elegans osm-3 kinesin gene in chemosensory neurons open to the external environment. I Mol Biol 247: 377-389.

Troemel ER, Chou JH, Dwyer ND, Colbert HA, Bargmann CI. 1995. Divergent seven transmembrane receptors are candidate chemosensory receptors in C. elegans. Cell 83: 207218.

Troemel ER, Kimmel BE, Bargmann CI. 1997. Reprogramming chemotaxis responses: Sensory neurons define olfactory preferences in C. elegans. Cell 91: 161-169.

Troemel ER, Sagasti A, Bargmann CI. 1999. Lateral signaling mediated by axon contact and calcium entry regulates asymmetric odorant receptor expression in C. elegans. Cell 99: 387-398. 
Tucker M, Sieber M, Morphew M, Han M. 2005. The Caenorhabditis elegans aristaless orthologue, alr-1, is required for maintaining the functional and structural integrity of the amphid sensory organs. Mol Biol Cell 16: 4695-4704.

van der Linden AM, Nolan KM, Sengupta P. 2007. KIN-29 SIK regulates chemoreceptor gene expression via an MEF2 transcription factor and a class II HDAC. EMBO J 26: 358-370.

van der Linden A, Wiener S, You YJ, Kim K, Avery L, Sengupta P. 2008. The EGL-4 PKG Acts with the KIN-29 SIK and PKA to regulate chemoreceptor gene expression and sensory behaviors in Caenorhabditis elegans. Genetics 180: 1475-1491.

Vosshall LB, Amrein H, Morozov PS, Rzhetsky A, Axel R. 1999. A spatial map of olfactory receptor expression in the Drosophila antenna. Cell 96: 725-736.

Wes PD, Bargmann CI. 2001. C. elegans odour discrimination requires asymmetric diversity in olfactory neurons. Nature 410: $698-701$. 


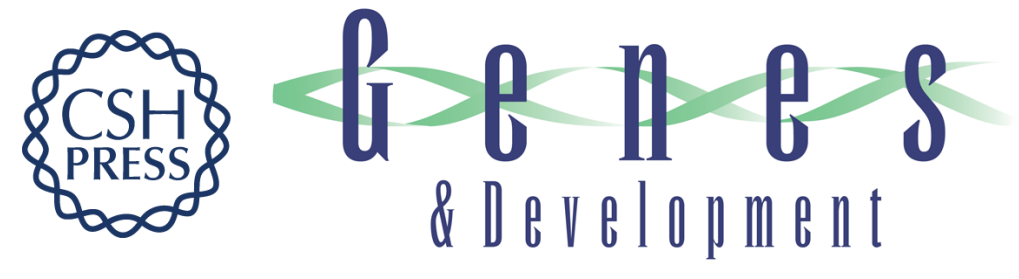

\section{The homeodomain protein $h m b x-1$ maintains asymmetric gene expression in adult $C$. elegans olfactory neurons}

Bluma J. Lesch and Cornelia I. Bargmann

Genes Dev. 2010, 24:

Access the most recent version at doi:10.1101/gad.1932610

Supplemental http://genesdev.cshlp.org/content/suppl/2010/08/11/24.16.1802.DC1
Material

References This article cites 72 articles, 28 of which can be accessed free at:

http://genesdev.cshlp.org/content/24/16/1802.full.html\#ref-list-1

License

Email Alerting Receive free email alerts when new articles cite this article - sign up in the box at the top

Service right corner of the article or click here.

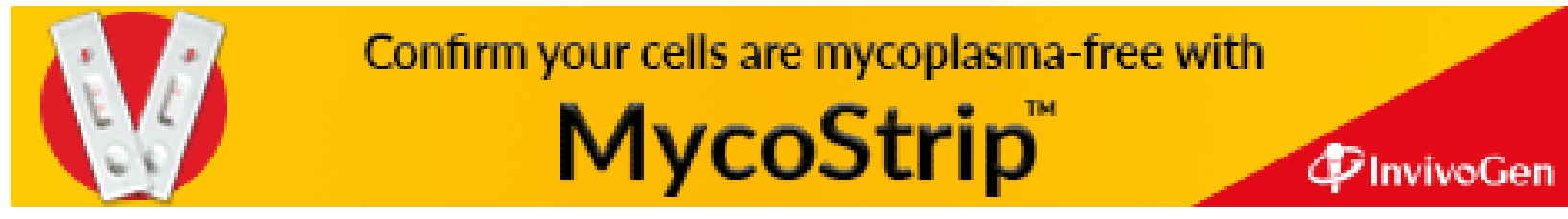

